\title{
The Effectiveness of Topical Metronidazole Management for Malignant Fungating Wound Odor: Nursing Perspective
}

\author{
Rabia Haddad $^{1 *}$, Haneen Taha ${ }^{2}$ and Fadi Fawares ${ }^{3}$ \\ ${ }^{1}$ Department of Nursing, King Abdullah University Hospital/Jordan University of science and Technology, Irbid, Jordan \\ ${ }^{2}$ Department of Nursing, Jordanian Royal Medical Services, Amman, Jordan \\ ${ }^{3}$ Department of Nursing, King Hussein Cancer Center, Amman, Jordan
}

${ }^{*}$ Corresponding author: Rabia Haddad, Department of Nursing, King Abdullah University Hospital/Jordan University of science and Technology, Irbid, Jordan; Email: rabiahaddad200@yahoo.com

Received: December 24, 2021; Accepted: December 31, 2021; Published: January 07, 2022

\begin{abstract}
Objectives: Malignant fungating wounds (MFW) defined as a wound that arise when cancerous cells invade the skin and metastasis to the surrounding blood and lymph vessel [1], Malignant wounds, also known as fungating tumors, tumor necrosis, ulcerative malignant wounds, or fungating malignant wound [2]. Moreover, The prevalence of MFW is (5-10\%) of patient who suffer from metastasis tumors, it's happened in age between 60-70 years during last 6 months, the MFW effect on this area of body: Breast (62\%), Head and Face (24\%), Groin and Genitals (3\%), Back (3\%), and other (8\%) [3]. MFW effect on both a physical and psychological for the patient, caregiver, and clinician. These wounds are often associated with pain, odor, bleeding, and an unpleasant appearance. The goals in the care of patients with malignant wounds include managing wound exudate, odor, bleeding and pain, preventing infection, and promoting the emotional wellbeing of the patient and family [4]. The purpose of this study to evaluate topical metronidazole management of MFW odor.
\end{abstract}

Methods: A search using search engines such as PubMed and Google scholar were used in finding articles related to the review topic.

Key findings: This review highlighted the malignant fungating wounds (MFW) are uncommon [5] but create major challenges for nurses concerned in cancer care [6]. Patient felt socially isolated because he or she embarrassed from odor and leakage at the wound site, needs to change dressing and clothes constantly, so became low self-confidence to interact with others, change relationship with friends and even didn't like to see wound for their families to keep good impression in their mind about her or his self. Represent the worst part of the patient's cancer; live in a body that cannot be trust, therefore become isolation considering that wound-related stigma. It's not only effect on patient well-being but as well effect on all dimensions of quality of life for their families. There both a physical and an emotional challenge for the patient, caregiver, and clinician.

Conclusions: Topical metronidazole has spread use in clinical practice around the world; it is inexpensive, easy to use, and easily available. Despite the lack of clinical studies to support the use of topical metronidazole in treatment of malodor MFW. Perhaps the lack of data is due to the fact that many studies are conducted with terminal patients or that fungating wounds are relatively rare. Although this review did not find strong scientific evidence supporting the efficacy of topical metronidazole in control of malodor MFW through randomized clinical studies, this review provides the best available evidence to support the use of topical metronidazole in the treatment of patients with malodor MFWs. Health care provider can use topical metronidazole to manage malodor MFW, but additional researches in this area is still necessary.

Keywords: Malignant wound, Metronidazole, Fungating, Malodor

\section{Introduction}

Fungating wound complications result from disturb of blood and lymph vessels, causes hypoxia, edema and necrosis, so the patient suffer from Pain (31.2\%), followed by exudate (14.6\%), odor (10.4\%), itching (5.2\%), bleeding (4.2\%) [7], these symptoms MFWs often cause physical and psychological and social distress for the patients and their health care provider especially malodor, so patient with MFW feel embarrassment, and social isolation [8]. These malodor caused by the presence of necrotic tissue which is an ideal media for both aerobic and anaerobic bacteria, but mainly anaerobic bacteria that produce volatile fatty acids as a metabolic end product [9], this foul odor can cause gagging and vomiting and loss of appetite [10]. Exudate has a contributing factor to produce this malodor.

The Management of MFW is complex and challenging for health professionals especially control malodor [11]. Although there are multiple choices of dressings available to manage MFW odor, but suitable dressings to manage the odor still presents a challenge, therefore conduction of this review is to evaluate topical metronidazole management for MFW odor. 
In order to inspect the knowledge related to topical metronidazole for MFW odor management among cancer patients, holistic and comprehensive review were conducted using the electronic databases of CINAHL, Pub Med, and Medline included for articles published between 1990 and 2015.

\section{Malignant Wound Management}

After reading the title and abstract, lists of 29 articles were considered. After that we chose the articles which investigated topical metronidazole for MFW odor management among cancer patients. Although fungating wounds pose a challenge for patients and caregivers, there are limited researches articles have been published on this topic [12]. Only 6 research articles were included in this review. The 6 articles published from 1992 to 2014 were selected and formed the basis for this review. The initial study included was published in 1992, with most studies published in 2014. These paper were published in nursing and Medical journals.

The 6 studies that build this review were quantitative researchbased studies. 4 studies were randomized clinical trial studies, one study was descriptive cross-sectional studies, and one study was systematic review study. This systematic review included 1 randomized clinical trial study, 3 uncontrolled clinical trial studies, 1 case series study and 5 case report studies. Although only 6 studies were included in this research review, In the 6 studies a wide variety of designs, tools, and sample sizes were used.

MFW odor management remains insufficiently treated worldwide and still uncontrolled by evidence [13]. Until now approach regarding the most effective management protocol for patients with malodorous MFW is lacking [14]. However, based on this review topical metronidazole benefited patients with MFW, relieving them from their distressing malodor without fear of adverse reactions can be concluded.

\section{The Effectiveness of Metronidazole in Wound Care Management}

Metronidazole (Flagel) is a synthetic drug in the pharmacologic drug class Nitroimidazole. It prevents replication of bacteria by binding to their DNA [15]. It is an antibiotic agent used topically for reduction of malodor and is particularly effective for anaerobic bacteria and protozoa. While used widely there are variations in the concentrations used and the methods of application [16]. Numerous reports support its effectiveness in relieving malodor often within only a few days [17]. However topical Metronidazole may become ineffective when diluted by large amounts of exudates [18].

Topical metronidazole gel applied directly to the wound surface once or twice daily following cleansing, it is effective in the elimination or substantial reduction of malodor. Studies show it is usually effective within 2-3 days [19]. The moist environment produced by the gel may also help to promote wound debridement by facilitating autolysis of slough and necrotic tissue Metronidazole gel has been shown to be the best evidence-based practice for odour control. However, prolonged usage of antibiotics can become ineffective due to drug resistance.
Grocott P [20] argued that in large, heavily exuding wounds, topical application of gel might be ineffective as it becomes diluted by the exudate, the gel being absorbed by the dressings, whether the gel can penetrate deep enough into the necrotic tissue and the size of the wound may limit its efficacy. Also he argued that how the dose of topically applied gel is calculated, and recommend that more research is needed to support its use.

A double-blind placebo-controlled trial was conducted [21] to assess the value of topical metronidazole gel preparation in the palliation of the offensive odor of fungating tumors. During 11 days 9 patients with malodorous MFW were randomized into either control group 5 patients or treatment group topical Metronidazole gel 4 patients. Subjective odor assessments were performed by both patients and medical staff. This experiment confirms the efficacy of $0.8 \%$ metronidazole gel in reducing the odor associated with open fungating tumors without adverse effects. Recommendations from Bower et al for the control of malodor were daily wound cleansing and dressing for 7 days, followed by a 5-day course of metronidazole gel applied once daily at a dose of $1 \mathrm{~g} / \mathrm{cm}^{2}$.

Others studied [22] the use of topical metronidazole gel on 47 patients, and found a decrease in malodor in $95 \%$ of patients by day 14. Anaerobic infection was eliminated in $84 \%$ of patients. However, estimated that the cost of topical metronidazole was 10 times the cost of tablets. A significant reduction in pain, exudate and cellulites with the use of topical metronidazole also recorded.

Kuge et al. [23] conducted a microbiological assessment and measured sensitivity to metronidazole gel $0.8 \%$ in 5 female breast cancer patients with fungating wound. In this uncontrolled clinical study, the authors determined that after the odor was gone after 2-5 days, Culture of swabs show a decrease or disappearance of anaerobic colonies. Adverse reactions of metronidazole did not occur, although this uncontrolled study reported the expected results (odor elimination).

Gethin et al. conducted an international descriptive crosssectional on-line survey [24-28] to determine the current practice in the management of wound odor. A specific questionnaire in English, Spanish, Italian and German was emailed to wound care organizations worldwide, palliative and oncology nursing organizations. 1444 people from 36 countries responded. $46.7 \%$ of respondents encounter patients with MFW on a monthly basis around 811 patient. Topical Metronidazole gel was one of current practice in management of MFW odor that used by $56.9 \%, 87.9 \%$ of them reported this as being 'somewhat effective' or 'very effective'. In this study large numbers who did respond from a range of health care settings support using of metronidazole gel for MFW odor management.

Another topical form of Metronidazole is tablets that might be crushed and mixed with sterile water to create either a $0.5 \%$ solution $(5 \mathrm{mg} / \mathrm{cc}$ ) or $1 \%$ solution. This is then used as a wound irrigate or a gauze is soaked in the solution and applied to the wounds. There is much anecdotal evidence to support this practice although little scientific evidence exists. 
Lian et al. in a prospective randomized experimental study, comparing effectiveness of green tea versus topical Metronidazole powder in controlling malodor of MFW. 30 patients with malodorous MFW were randomized into either treatment green tea 15 patients or control group topical Metronidazole powder15 patients. All patients in both groups showed reduction in odor control by Day 7 without significant difference, this study give heath care provider alternative cost effective treatment for MFW. Metronidazole powder is used more often than metronidazole gel because it is very costly.

A systematic review of topical treatment to control MFWs odor done by Da Costa Santos et al. found that Metronidazole was cited in 10 studies The interventions ranged from the topical application of a gel or solution of Metronidazole in concentrations of $0.75 \%-0.8 \%$ to the treatment of the MFWs with crushed metronidazole tablets; on average, application took place once a day, and treatment lasted for 14 consecutive days. It found in this review that this treatment known in clinical practice to be effective for the control of MFW odor.

\section{Conclusion}

Topical metronidazole has spread use in clinical practice around the world; it is inexpensive, easy to use, and easily available. Despite the lack of clinical studies to support the use of topical metronidazole in treatment of malodor MFW. Perhaps the lack of data is due to the fact that many studies are conducted with terminal patients or that fungating wounds are relatively rare. Although this review did not find strong scientific evidence supporting the efficacy of topical metronidazole in control of malodor MFW through randomized clinical studies, this review provides the best available evidence to support the use of topical metronidazole in the treatment of patients with malodor MFWs. Health care provider can use topical metronidazole to manage malodor MFW, but additional researches in this area is still necessary.

\section{Implications in Nursing Practice}

This review paper will add basic knowledge regarding management of odor with Malignant Fungating Wound. Improve quality of life for cancer patient during illness trajectory, Improve social, psychological, and spiritual life for them. Help care provider to broaden knowledge about effectiveness of metronidazole. Help other researcher to study other clinical variable.

\section{Declarations}

\section{Conflict of Interest}

The Author(s) declare(s) that they have no conflicts of interest to disclose.

\section{Funding}

This research received no specific grant from any funding agency in the public, commercial or not-for-profit sectors.

\section{Authors' Contributions}

Rabia H, Fadi F. initiated and designed the review and formulated the first draft of the manuscript. Haneen T. critically reviewed the final manuscript.

\section{References}

1. Probst S, Arber A, Faithfull S (2013) Malignant Fungating Wounds-The Meaning of living In an Unbounded Body. European Journal of Oncology Nursing 17: 38-45.区

2. Ferrell, Coylle, Paice, (2014). Oxford textbook of palliative Nursing, $4^{\text {th }}$ ed. Oxford University Press.

3. Dowsett C (2002) Malignant fungating wounds: assessment and management. British Journal of Community Nursing 7: 394-400. [crossref]

4. Ferrell B, Coyle N (2010) Oxford textbook of palliative Nursing, $3^{\text {rd }}$ ed., Oxford University Press, New York.

5. Naylor WA (2002) A guide to wound management in palliative care. International Journal of Palliative Nursing 11: 572-557. [crossref]

6. Lo SF, Hu WY, Hayter M, Chang SC, Hsu MY, et al. (2008). Experiences of living with a malignant fungating wound: a qualitative study. Journal of Clinical Nursing 17: 2699-2708. [crossref]

7. Graves ML, Sun V (2013) Providing Quality Wound Care at the End of Life. Journal of Hospice \& Palliative Nursing 15: 66-74

8. DaCSantos CM, De Mattos PCA, Nobre MRC (2010) A Systematic Review of Topical Treatments to Control the Odor of Malignant Fungating Wounds. Journal of Pain and Symptom Management 39: 1065-1076. [crossref]

9. Clark, Jane (2002) Metronidazole gel in managing malodorous fungating Wound. British Journal of Nursing 11: 54-56.

10. O’Brien C (2012) Malignant wounds Managing odour. Canadian Family Physician 58: $272-274$

11. Lian S, XuY, Goh S, Aw F (2014) Comparing the Effectiveness of Green Tea verus topical Metronidazole Powder in Malodorous Control of Fungating Malignant wounds in a Controlled Randomized Study. Proceedings of Singapore Healthcare 23: 3-9.

12. Bergstrom KJ (2011) Assessment and Management of Fungatin Wounds. Journal of Wound Ostomy \& Continence Nursing 38: 31-37. [crossref]

13. Grocott P, Gethin G, Probst S (2013) Malignant wound management in advanced illness: new insights. Current Opinion in Supportive and Palliative Care 7: 101-105. [crossref]

14. Hampton S (2008) Malodorous fungating wounds: how dressings alleviate symptoms. British Journal of Community Nursing 13: sup. 31-38.

15. Alexander S (2009) Malignant Fungating Wounds: Epidemiology, A etiology, Presentation and Assessment. Journal of Wound Care 18: 273-276.

16. Gethin G (2010) Managing malodour in palliative care wounds in primary care. Nursing in General Practice.

17. Alexander S (2009) Malignant fungating wounds: managing malodour and exudate. Journal of Wound Care 18: 374-382. [crossref]

18. Lazelle-Ali C (2007) Psychological and Physical Care of Malodorous Fungating Wounds. British Journal of Nursing 16: 19-20.

19. Draper $\mathrm{C}$ (2005) The management of malodour and exudate in fungating wounds. British Journal of Nursing 14: 4-12.

20. Grocott $\mathrm{P}$ (1999) The management of fungating wounds. Journal of Wound Care 8: 232-234.

21. Bower M, Stein R, Evans TRJ, Hedley A, Pert P, et al. (1992) A double-blind study of the efficacy of metronidazole gel in the treatment of malodorous fungating tumours. European Journal of Cancer 28: 888-8. [crossref]

22. Finlay IG, Bowszyc J, Ramlau C, Gwiezdzinski Z (1996) The effect of topical 0.75\% metronidazole gel on malodorous cutaneous ulcers. Journal of Pain and Symptom Management 11: 158-162. [crossref]

23. Kuge S, Tokuda Y, Ohta M, Okumura A, Kubota M, et al. (1996). Use of metronidazole gel to control malodor in advanced and recurrent breast cancer. Japanese Journal of Clinical Oncology 26: 207-210. [crossref]

24. Gethin G, Grocott P, Probst S, Clarke E (2014) Current practice in the management of wound odour: An international survey. International Journal of Nursing Studies 51: 865-874. [crossref] 
Rabia Haddad (2022) The Effectiveness of Topical Metronidazole Management for Malignant Fungating Wound Odor: Nursing Perspective

25. Adderley UJ, Holt IG (2014) Topical Agents and Dressings for Fungating Wounds. The Cochrane Library 2. [crossref]

26. American Cancer Society (2009). Cancer Facts \& Figures for Hispanics.
27. Gibson S, Green J (2013) Review of Patients` Experiences With Fungating Wounds and Associated Quality Of Life. Journal of Wound Care 22: 265-275. [crossref]

28. Selby T (2009). Managing Exudate in Malignant Fungating Wounds and Solving Problems for Patients. Nursing Times 105: 14-7 [crossref].

Haddad R, Taha H, Fawares F (2022) The Effectiveness of Topical Metronidazole Management for Malignant Fungating Wound Odor: Nursing Perspective. Cancer Stud Ther J Volume 7(1): 1-4. 